\title{
Position-Dependent Extension of $\pi$-Conjugation in D- $\pi$-A Dye Sensitizers and the Impact on the Charge-Transfer Properties
}

\author{
Mateusz Wielopolski, ${ }^{\dagger} \dagger$ Jong-Hyung Kim, ${ }^{\ddagger}$ Young-Sam Jung, ${ }^{\ddagger}$ Young-Jae Yu, ${ }^{\ddagger}$ Kwang-Yol Kay, ${ }^{*} \S$
} Thomas W. Holcombe, ${ }^{\dagger}$ Shaik M. Zakeeruddin, ${ }^{\dagger}$ Michael Grätzel, ${ }^{\dagger}$ and Jacques-E. Moser*, ${ }^{\dagger}$

${ }^{\dagger}$ Laboratory for Photonics and Interfaces, Institute of Chemical Sciences and Engineering, Swiss Federal Institute of Technology, $\mathrm{CH}-1015$ Lausanne, Switzerland

${ }^{\ddagger}$ SolarSys Co. Ltd. BI Center and ${ }^{\S}$ Department of Molecular Science and Technology, Ajou University, Suwon 443-749, Korea

\section{Supporting Information}

ABSTRACT: A series of five organic donor- $\pi$-bridge-acceptor (D- $\pi$-A) sensitizers is investigated within the context of their photoinduced charge-transfer properties. Thereby, the focus is set on the impact of structural modifications of the molecular architecture on the $\pi$-systems of the dyes. In particular, two different modes of systematic extension of the sensitizers' $\pi$-systems, namely, (i) within the electron donating site and (ii) within the $\pi$ bridge, are investigated by means of steady-state and time-resolved spectroscopic methods. The photophysical studies of the molecules in solution and as deposited on $\mathrm{Al}_{2} \mathrm{O}_{3}$ or $\mathrm{TiO}_{2}$ films reveal that different effects on the charge-transfer characteristics evolve dependent where - within the molecular structure - the modification of the $\pi$-system is performed. Hence, $\pi$-extension of the donor sites, for instance, leads to a strong red shift of the absorption features and a variation of light-harvesting properties. Modifying the $\pi$-bridges results in a spatial decoupling of the HOMO and LUMO orbitals, which goes along with changes of the electronic coupling to $\mathrm{TiO}_{2}$. Furthermore, solution studies show that the electronic structure of the dyes governs their singlet excited-state features. As shown, the results obtained from these studies then allow important predictions about the deactivation of the excited states of these molecules adsorbed on $\mathrm{TiO}_{2}$. Finally, quantum chemical methods - among others, time-dependent density functional theory calculations - provide conclusive insight into the relationship between the electronic structure of the dyes and its impact on the photoinduced charge-transfer characteristics.

\section{INTRODUCTION}

The advantages of metal-free organic sensitizers, for the application in dye-sensitized solar cells (DSSCs), place them more and more into the focus of sensitizer research and development. Superior to their ruthenium-containing alternatives ${ }^{1}$ are their extinction coefficients and the nearly unlimited possibilities of varying parameters such as the overall $\pi$-conjugation, the distance between the donor and the acceptor, and the nature of the anchoring groups by wellestablished synthetic methods. ${ }^{2}$ This allows for fine-tuning the spectral and electronic features as well as for a control over charge-transfer kinetics and electronic coupling to $\mathrm{TiO}_{2}$. Understanding the influence of structural variations to the sensitizer, such as the length of $\pi$-conjugation or anchoring/ acceptor group choice on the DSSC device performance, is crucial for advancing this field and technology. ${ }^{3,4}$ Critical factors that influenced performance properties are (i) the excited-state redox potentials, which should be properly aligned with the conduction band of $\mathrm{TiO}_{2}$, (ii) the light-harvesting features of the sensitizer, (iii) the conjugation across the donor and anchoring groups, and (iv) the electronic coupling between the lowest unoccupied molecular orbital (LUMO) and the conduction band of the $\mathrm{TiO}_{2}{ }^{5}$ It has been shown that a sensitive interplay between all of these factors governs a vectorial and efficient electron flow from the electron donating moiety of the dye toward the semiconductor surface. Most of these studies, however, concentrate on the variation of one parameter at a time. The anchoring groups have been varied within one very similar system, ${ }^{6}$ the $\pi$-conjugation, ${ }^{7}$ or the distance between the donor and the acceptor. ${ }^{8}$

We wish to report on the systematic extension of the $\pi$ system by introducing thiophene units at various positions of the dye architecture. We believe that the impact of such structural changes on the charge-transfer properties of the dyes varies according to the modified site within the dye architecture. Hence, it is possible to influence the chargetransfer properties differently by extending the $\pi$-conjugation at different positions of the dyes. Throughout our investigations, we mainly focus on monitoring the charge-transfer kinetics between the dye and $\mathrm{TiO}_{2}$ and correlate them with the structural changes and the electronic properties of the dyes.

Starting with SD-1 as a reference, two strategies were followed to extend the $\pi$-system. First, the distance between the electron donor moiety and the anchoring group was increased

Received: March 9, 2013

Revised: June 11, 2013

Published: June 12, 2013 
Chart 1. Molecular Structures of SD Dyes ${ }^{a}$

SD-1
SD-2

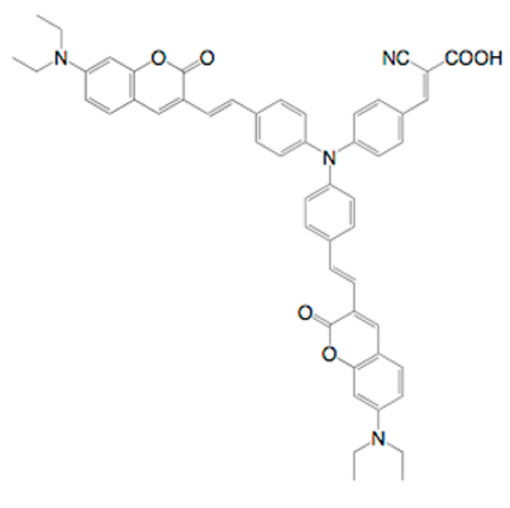

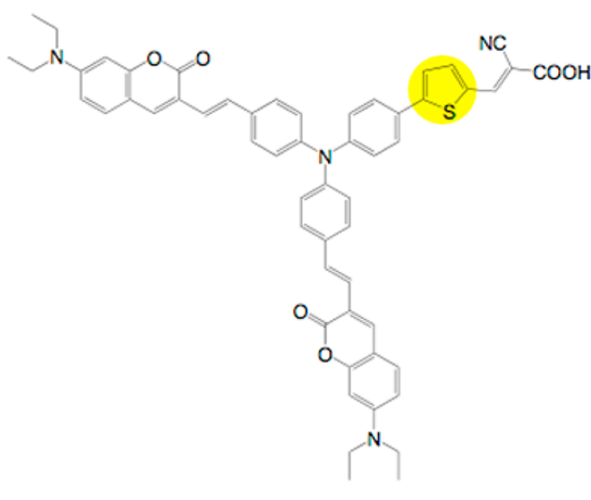

SD-3

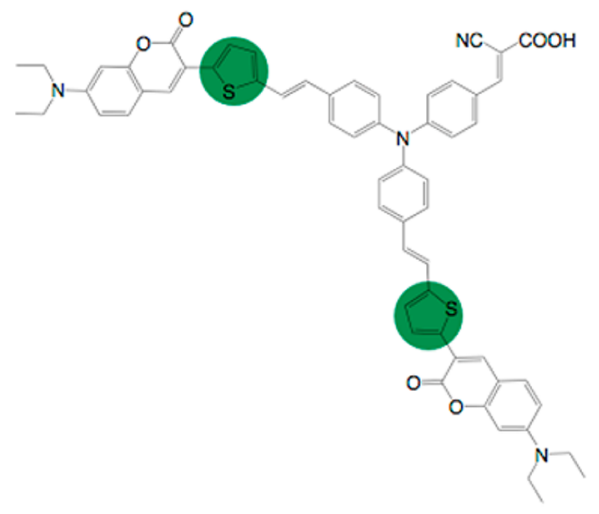

SD-5

SD-4
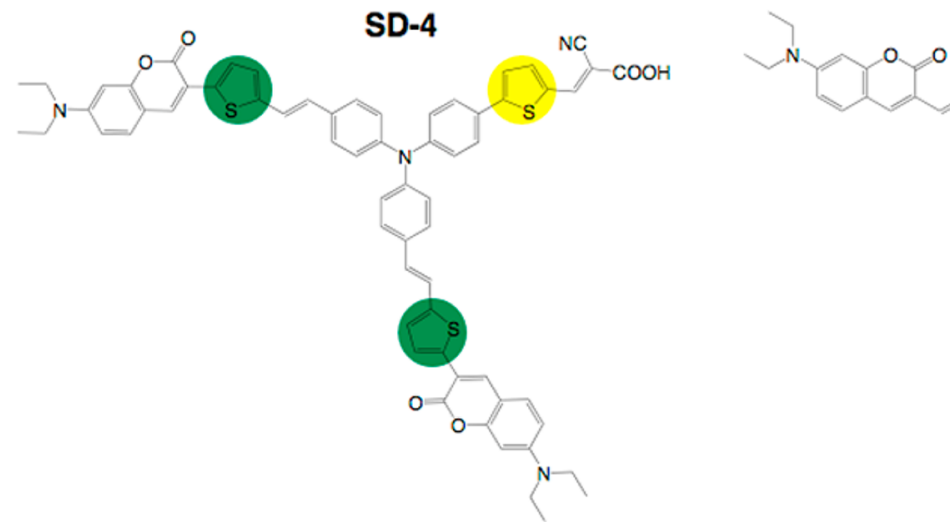

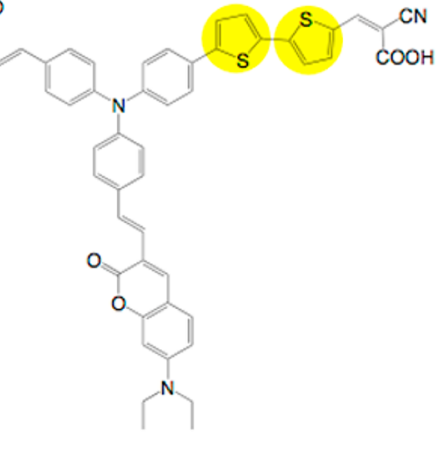

${ }^{a}$ Circles indicate the extension of the $\pi$-system of the linker (yellow) and the $\pi$-system of the donor moiety (green).

by inserting one and two thiophenes between the triphenylamine (TA) and cyanoacrylic acid to yield SD-2 and SD-5, respectively. The second strategy involves the extension of the donor $\pi$-system, that is, SD-1 versus SD-3. Finally, in SD-4, both strategies were combined, that is, extension of the donoracceptor distance and the $\pi$-system of the donor (Chart 1 ).

The photovoltaic performances of SD-1, SD-2, and SD-5 have already been reported before. ${ }^{9}$ It has been proposed that in addition to the red shift of the spectral response caused by inserting the thiophene units, their insertion favors the creation of charge recombination centers. Nanosecond flash photolysis studies of SD-1, SD-2, and SD-5, supported this contention, showing significant differences of the charge recombination rate constants with the insertion of thiophenes between the donor and acceptor. Regarding the photovoltaic performance, the $J_{\mathrm{sc}}$ values also increased with insertion of the thiophenes, whereas the $V_{\text {oc }}$ values decreased. To obtain a better understanding of these effects, we decided to include SD-3 and SD-4 and further investigate further the role of additional conjugation and the placement of this conjugation as an expansion of this dye series. This allows us to concentrate our studies on the impact of particular structural changes to the chemical architecture on the charge injection and charge recombination of this sensitizer class into and from $\mathrm{TiO}_{2}$. The photophysical investigation of the entire SD series allowed, for the first time, a comparison of the two different spatial options for extending the $\pi$-system of the dyes: extension between the donor and acceptor as well as purely extending the donor conjugation. These results permit us to address specific structural parameters within this particular dye architecture and to learn more about how the placement of extended conjugation affects charge injection/ recombination processes within these devices. Therefore, we focus rather on the photophysical features of the dyes than on their performance within working DSSC devices. Extensive spectroscopic - steady-state and time-resolved - as well as theoretical studies were employed to elucidate the photophysical properties of the dyes and their structure-dependent interactions with $\mathrm{TiO}_{2}$.

\section{RESULTS AND DISCUSSION}

Synthesis. The synthesis of SD-1, SD-2, and SD-5 was reported elsewhere. The dyes SD-3 and SD-4 were synthesized according to the steps depicted in Scheme 1. Every step of the reaction sequence proceeded smoothly and efficiently to give a good or moderate yield of the product. (See the Experimental Section in the Supporting Information for the synthetic details.) The aldehydes $\mathbf{3}$ and $\mathbf{5}$ were synthesized by the Heck reaction using $\mathrm{Pd}(\mathrm{OAc})_{2}$ as catalyst. Compounds 3 and 5 were then reacted with cyanoacetic acid in the presence of piperidine to produce the dyes SD-3 and SD-4, respectively.

Absorption Studies. The absorption spectra of all dyes in $0.1 \mathrm{mM}$ dimethylformamide (DMF) solution and after adsorption on $3 \mu \mathrm{m}$ thick mesoporous $\mathrm{TiO}_{2}$ films are shown in Figure 1 and Figure $S 1$ in the Supporting Information, respectively.

In solution, all dyes exhibit a broad absorption maximum between 380 and $550 \mathrm{~nm}$. In accordance with the $\pi$-conjugated donor-acceptor type chemical architecture of all dyes, these maxima correspond to $\pi-\pi^{*}$ intramolecular charge-transfer transitions. The molar extinction coefficients do not vary 
Scheme 1. Synthesis of SD-3 and SD-4 ${ }^{a}$
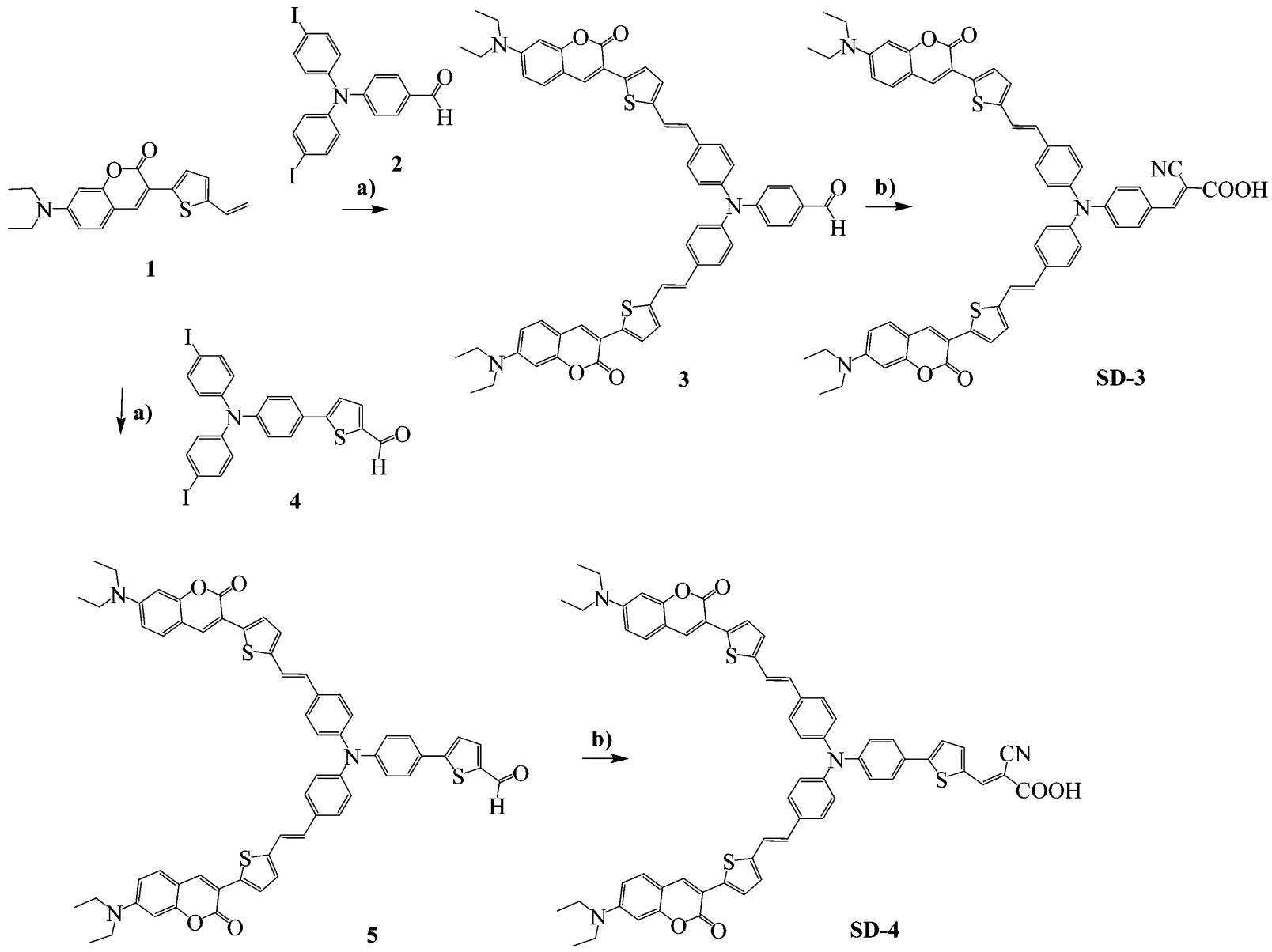

${ }^{a}(\mathrm{a}) \mathrm{Pd}(\mathrm{OAc})_{2}, \mathrm{Bu}_{4} \mathrm{NBr}, \mathrm{K}_{2} \mathrm{CO}_{3}, \mathrm{DMF}, 95^{\circ} \mathrm{C}, 16 \mathrm{~h}, 68.5 \%$ for 3, 66.9\% for 5. (b) Cyanoacetic acid, piperidine, chloroform, reflux, $16 \mathrm{~h}, 63.8 \%$ for SD-3, 78.1\% for SD-4.

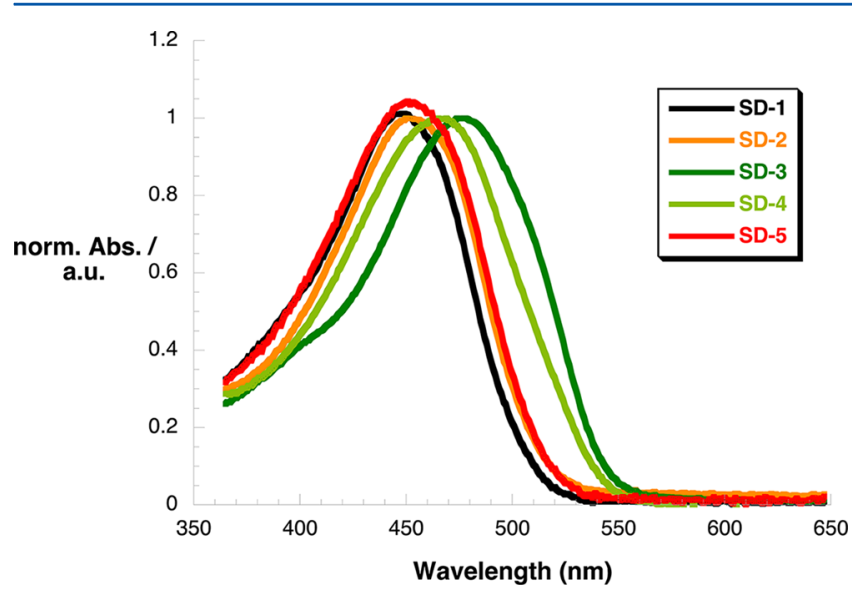

Figure 1. Normalized absorption spectra of the SD dyes as $0.1 \mathrm{mM}$ DMF solutions.

significantly within the dye series and have been measured in the range of $10^{4} \mathrm{M}^{-1} \mathrm{~cm}^{-1}$. The red shift of the absorption clearly depends on the position of the inserted thiophenes. As compared with SD-1, in SD-2 and SD-5 the red shift in DMF is $5 \mathrm{~nm}\left(202 \mathrm{~cm}^{-1}\right)$ upon insertion of the first thiophene and only $6 \mathrm{~nm}\left(242 \mathrm{~cm}^{-1}\right)$ upon inserting the second thiophene. Hence, the second thiophene contributes to the broadening the spectral breadth to a much lesser extent than the first, and the main impact of this second thiophene is to increase the donor-acceptor distance.

The situation is different when considering SD-3 and SD-4. Here the thiophenes - as part of the donor moieties - impact the spectral shift of the dyes to a much greater extent and lead to red shifts of $30\left(1335 \mathrm{~cm}^{-1}\right)$ and $20 \mathrm{~nm}\left(972 \mathrm{~cm}^{-1}\right)$ for SD3 and SD-4, respectively. Interestingly, the insertion of the thiophene into the $\pi$-spacer of SD-4 effects a blue-shift compared with SD-3. As shown in the theoretical studies, the distribution of the electron density of the LUMO in SD-4 is much more localized than in SD-3, which in turn affects the absorption spectrum. The spectral features after adsorption on $\mathrm{TiO}_{2}$ (Figure $\mathrm{S} 1$ in the Supporting Information) follow the trends as established in solution with slightly smaller differences. The absorption edges of all dyes are shifted to the blue by $\sim 30 \mathrm{~nm}\left(1074 \mathrm{~cm}^{-1}\right)$. This shift of the absorption to higher energies on $\mathrm{TiO}_{2}$ may be ascribed to $\mathrm{H}$-aggregation and the deprotonation of the carboxylic acid. ${ }^{10}$ This effect appears to be strongest for SD-5.

Molecular Modeling. To further understand how the modifications of the electronic properties were affected by the variation of the chemical structure of the dyes, we have employed theoretical calculations at the density functional level of theory. The geometries of all structures were optimized using the $\mathrm{M} 062 \mathrm{X}^{11}$ functional with the $6-31 \mathrm{G}^{* 12}$ basis sets, as implemented into the Gaussian $09^{13}$ suite of programs. 
SD-1
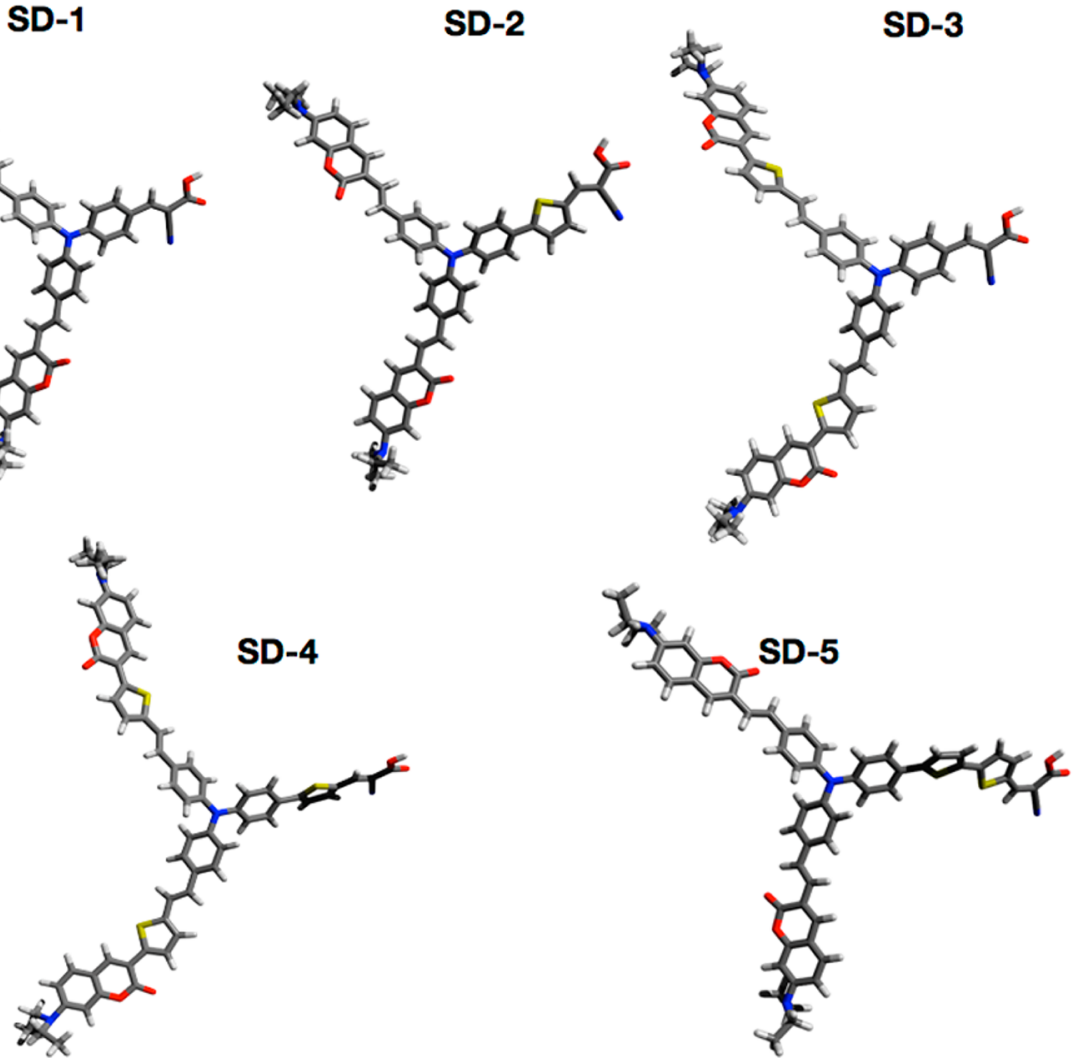

Figure 2. Energy-minimized ground-state geometries of all SD dyes as computed using the M062X/6-31G* DFT method.

The influence of the position of the insertion of the thiophenes is already visible when considering the ground-state geometries of the dyes (Figure 2). Taking SD-1 as a reference, it is seen that the insertion of the thiophene into the spacer between donor and acceptor induces a twist between the phenyl ring of TA and the anchoring group. In SD-1, the anchor is coplanar with the TA moiety, whereas dihedral angles of 27 and $12^{\circ}$ are found in SD-2 and SD-5, respectively. Furthermore, the inserted thiophenes are not coplanar with the phenyl ring (SD-2) and with each other (SD-5). Considering SD-3 and SD-4, the insertion of the thiophenes into the donor moiety leaves the aromatic chains planar and rather extends the $\pi$-conjugation in the donor part of these two dyes. Representations of the frontier orbitals (Figure 3) corroborate this assumption.

To calculate the frontier orbitals and excited-state features of the dyes, time-dependent (TD) DFT methods have been employed. The TD-DFT calculations were conducted on the aforementioned optimized geometries using the M062X functional and the $6-31 \mathrm{G}^{*}$ basis set. For each molecule, the ten lowest excited states have been computed by applying the polarizable continuum model ${ }^{14}$ to simulate solvent interactions in dichloromethane.

Considering the frontier orbital schemes, Figure 3 shows the two highest-occupied (HOMOs) and the three LUMOs because these are the orbitals involved in the main excitations of the molecules. Again, SD-1 will be considered as reference. The HOMO is well-delocalized over the entire molecular structure, whereas the electron density of the HOMO-1 clearly shifts away from the anchoring part. Upon inserting the thiophenes into the donor in SD-3 and SD-4, the delocalization of the orbital coefficients is extended onto the thiophene rings, and the HOMO energies increase by 0.08 and $0.19 \mathrm{eV}$ for SD-3 and SD-4, respectively, which facilitates the oxidation of these two dyes as compared with SD-1. Equally, the delocalization of the HOMO-1 in SD-3 and SD-4 increases as well, which significantly raises their energies by more than $0.3 \mathrm{eV}$ as compared with SD-1. The two lowest computed excited states (Table S1 in the Supporting Information) suggest that it is these two orbitals that in the oxidized state accommodate the positive charge. Hence, in SD-3 and SD-4, the hole can be stabilized more efficiently due to the enhanced delocalization. Furthermore, the LUMO+1, which is localized on the donating sites and comprises the main contributions to the excitations with the highest oscillator strengths, drops in energy by 0.20 and $0.18 \mathrm{eV}$ for SD-3 and SD-4, respectively, in comparison with the other dyes.

Inserting the thiophenes in the spacer between donor and acceptor induces different effects, which is, in particular, discernible in the LUMO representations of Figure 3. In SD1 and SD-3, the LUMO is delocalized from the anchoring part entirely into the donor-site of the molecules. In SD-2, SD-4, and SD-5, they are localized on the accepting moieties. Obviously, such a localization of the electron density on the spacer and anchoring groups results in lowering of the LUMO energies by $0.25,0.26$, and $0.38 \mathrm{eV}$, as seen for SD-2, SD-4, and SD-5, respectively. The decoupling of the HOMOs and LUMOs is most likely due to the loss of planarity induced by the thiophenes in the spacer. This is further accompanied by significant lowering of the HOMO-LUMO energy gap owing to the increase in $\pi$-conjugation length. This situation prompts to a typical donor-acceptor type dye architecture in SD-2, SD4, and SD-5. As a consequence, photoinduced excited-state formation effects a vectorial shift in charge density from the 


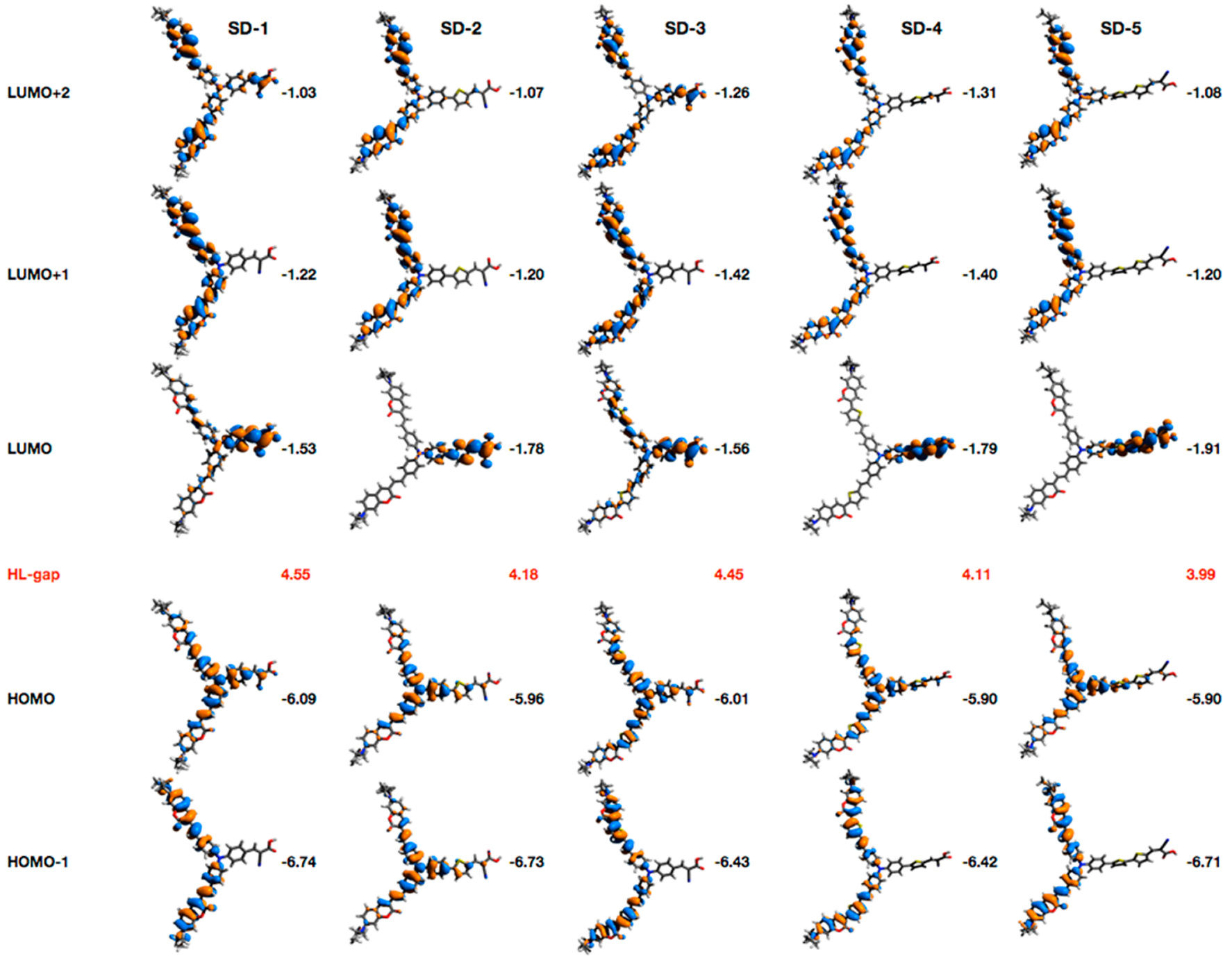

Figure 3. Frontier orbital representations with the corresponding energies and HOMO-LUMO energy gaps of all SD dyes as computed using the M062X/6-31G* TD-DFT method in simulated dichloromethane environment.

donor to acceptor, which implies an intramolecular radical ion pair formation, as confirmed by transient absorption studies of DMF solutions (see later). In SD-1 and SD-3, the excited states are characterized by rather delocalized excitations of one electron within the strongly overlapping HOMO and LUMO electron density cloud. Important in this context, the LUMO+1 in SD-3 and SD-4 is shifted to lower energies by more than 0.2 $\mathrm{eV}$ due to the extension of the donor conjugation by the thiophenes. This particularly influences the excited-state character of SD-3, where the LUMO+1 energy approaches the LUMO level by $0.14 \mathrm{eV}$. In the other dyes, the energy difference between the LUMO and LUMO+1 constitutes at the minimum $0.31 \mathrm{eV}$, which is more than twice as much as in SD3.

For SD-5, the orbital representations suggest the strongest decoupling of the LUMO and the longest $\pi$-conjugation path, which is evident in its lowest HOMO-LUMO gap. Thus, the electronic structure in SD-5 is comparable to the one in SD-2 and SD-4, which explains the similar photophysical behavior of SD-2, SD-4, and SD-5 (see below). In SD-1 and SD-3, the LUMO coefficients extend deeply into the donor sites, and this appears to be less favorable due to the higher energies of these orbitals and the increasing HOMO/LUMO energy gaps.
Scrutinizing the computed excited states of the dyes gives further insights into the influence of the thiophenes at different positions of the dye architecture. Table S1 in the Supporting Information summarizes the five lowest excited states including the contributions of the most significant orbital transitions. Obviously, the lowest energy excitations in all compounds can be assigned to HOMO $\rightarrow$ LUMO transitions with relatively high changes of the dipole moments between 20 and $30 \mathrm{D}$. With increasing conjugation length, contributions from lower HOMOs such as HOMO-1 and HOMO-2 become significant for the excitations as well. The corresponding energies and excitation wavelengths of the lowest transitions match well with the outcome of the absorption studies in solution and represent the trends in the HOMO-LUMO energy gaps. Thereby, the oscillator strengths increase from SD-1 to SD-2 to SD-5 to SD4 to SD-3, suggesting the highest extinction coefficients for SD4 and SD-3. Considering the second excited states, although higher in energy, the oscillator strengths and dipole moments $(30-40$ D) exceed the values of the first excited state. Remarkably, in SD-3 and SD-4 the energies of the second excitation differ by $<0.1 \mathrm{eV}$ compared with the first excited state. Furthermore, they are significantly shifted to the red in contrast with SD-1, SD-2, and SD-5. Regarding the fact that 
the main contributions to the second excited states stem from a $\mathrm{HOMO} \rightarrow \mathrm{LUMO}+1$ transition, this is well-explained by the significantly lower LUMO+1 level in these two dyes. In other words, the insertion of the thiophenes into the donor impacts the absorption cross sections of SD-3 and SD-4 to a much higher extent than in the remaining dyes. Additionally, as seen in Figure 3 the energetic proximity of the LUMO and LUMO +1 in SD-3 suggests the strongest mixing of the first two excitations. Because of the significantly lower oscillator strengths of the higher excited states, their contributions to the optical properties may be neglected.

On the basis of these results, it is safe to assume that by inserting the thiophenes into the spacer between the TA and the cyano acrylic acid it is possible to extend the $\pi$-conjugation length and lower the energies of the HOMO to LUMO excitations, which in all dyes constitute the energetically lowest excited states. Inserting the thiophenes into the donor has a strong effect on the LUMO+1 and hence on the second excited state, allowing for a mixing between the first and the second excitations in SD-3 and SD-4. Conclusively, the singlet excitations are directly correlated with the $\pi$-system architecture of the dyes, so that even slight changes of the chemical structure will have an impact on the absorption properties.

Noticeably, decoupled HOMO/LUMO orbitals and lower energy gaps most likely increase the radical ion pair character of the singlet excited states, leading to facilitated intramolecular electron transfer between localized states. As a consequence, the spectral signatures of the TA radical cation will broaden and shift to the red, as seen throughout the transient absorption studies.

In summary, the theoretical calculations show the different effects of structural changes performed on two different sites of these donor- $\pi$-spacer-acceptor type dyes. The results imply that the $\pi$-conjugation may be influenced by $\pi$-extension of either the donor or the $\pi$-spacer, leading to two main types of electronic transitions. The relative intensity and energy of these transitions depends on the electronic structure of the molecules and impacts their spectral and charge transfer properties.

Time-Resolved Spectroscopy. To probe the chargetransfer kinetics of the dyes and their dependence on the structural variations, we have employed time-resolved spectroscopic techniques. Apart from investigating the dyes asdeposited on $3 \mu \mathrm{m}$ thick $\mathrm{TiO}_{2}$ films, further studies have been performed on $0.1 \mathrm{mM}$ DMF solutions of the dyes and asdeposited on $3 \mu \mathrm{m}$ thick $\mathrm{Al}_{2} \mathrm{O}_{3}$ films.

As confirmed by the TDDFT calculations (see above), singlet excitation leads to an intramolecular charge-transfer event: the HOMO-to-LUMO, $\pi-\pi^{*}$-excitation shifts charge density from the electron-donating TA moiety to the electronaccepting cyanoacrylic acid anchor/acceptor moiety. Additional orbital involvement, such as HOMO to LUMO+1 charge density shifts, also contributes. Figure 4 shows the spectral fingerprints after photoexcitation of SD-1 and SD-3. Characteristic for the formation of the charge-separated (CS) state is a negative feature below $580 \mathrm{~nm}$, which is associated with the singlet bleach due to the absorption of the chromophore and a rather broad maximum in the region between 600 and $750 \mathrm{~nm}$, which corresponds to the absorption of the radical cation of TA. ${ }^{15}$ Both features vary in accordance with the extension of the $\pi$-system and depend on the position of the inserted thiophenes. In SD-3 (Figure 4) and SD-4, for instance, the bleaching is significantly shifted to the red by $50 \mathrm{~nm}$, as compared with SD-1, SD-2, or SD-5 (Figure 5). Hence, the

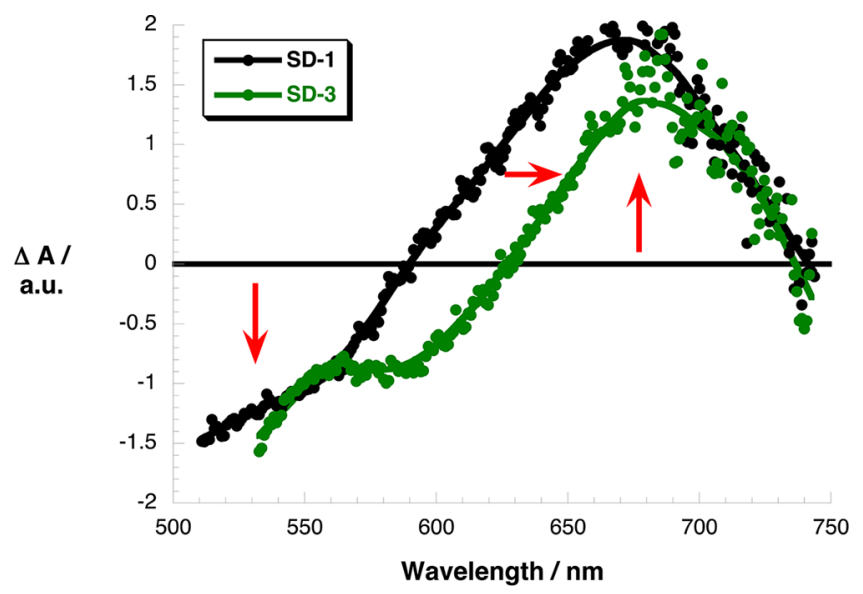

Figure 4. Spectral signatures of the singlet excitation in 0.1 mM DMF solutions of SD-1 (black) and SD-3 (green) resulting from femtosecond transient absorption studies at $480 \mathrm{~nm}$ excitation. The arrows indicate the shift of the corresponding signals upon structural modifications as mentioned in the text.

insertion of the thiophenes into the donor moiety directly affects the photoinduced absorption and leads to a bathrochromic shift of the spectral characteristics of SD-3 and SD4. On the contrary, the insertion of the thiophenes into the spacer between donor and acceptor does not influence the spectral positions of the maxima and minima of the photoexcited state, as seen from the comparison of the spectra of SD-1, SD-2, or SD-5 in Figure 5. Therefore, the modification of the chemical architecture has less impact on the spectral properties when placed between the donor and acceptor than when simply extending the donor $\pi$-conjugation length. The TDDFT calculations (see above) show that the increased donor $\pi$-conjugation changes the orbitals involved in the photoexcitation processes, given that the HOMO-to-LUMO and HOMO-to-LUMO+1 excitations play the major role in these systems. In particular, the LUMO+1 in SD-3 and SD-4 drops in energy due to the increased donor conjugation, which allows for a mixing between the HOMO $\rightarrow$ LUMO and $\mathrm{HOMO} \rightarrow \mathrm{LUMO}+1$ excitations. The mixing is reflected by the fact that the energy difference between the first and the second excited states is $<0.1 \mathrm{eV}$ in SD-3 and SD-4, whereas it exceeds $0.3 \mathrm{eV}$ for the other compounds.

From a comparison among SD-1, SD-2, and SD-5 (see Figure 5) a dependence of the kinetics on the distance between the donor and the acceptor is found. In SD-1, the singlet population (associated with the negative feature below $600 \mathrm{~nm}$ ) and the appearance of the radical cation signature occur simultaneously, which suggests that the charge density shifts in a coherent step, immediately on the time scale of the excitation. In SD-2 and SD-5, the bleach recovers faster with increasing number of thiophenes. This spatial separation of the donor and the acceptor decreases the coextensivity of the HOMO and the LUMO and increases the radical ion pair character of the resulting singlet excited state. As a consequence, the bleaching is less pronounced in SD-2 and SD-5 than the corresponding maximum of the TA radical cation for SD-1. The rate constants for the formation of the CS states $\left(k_{\mathrm{CS}}\right)$ have been determined from the rise of the cation signatures at $650 \mathrm{~nm}$. The corresponding time-absorption profiles have been approximated, chirp-corrected, and fitted exponentially to give rise times on the order of hundreds of femtoseconds. 

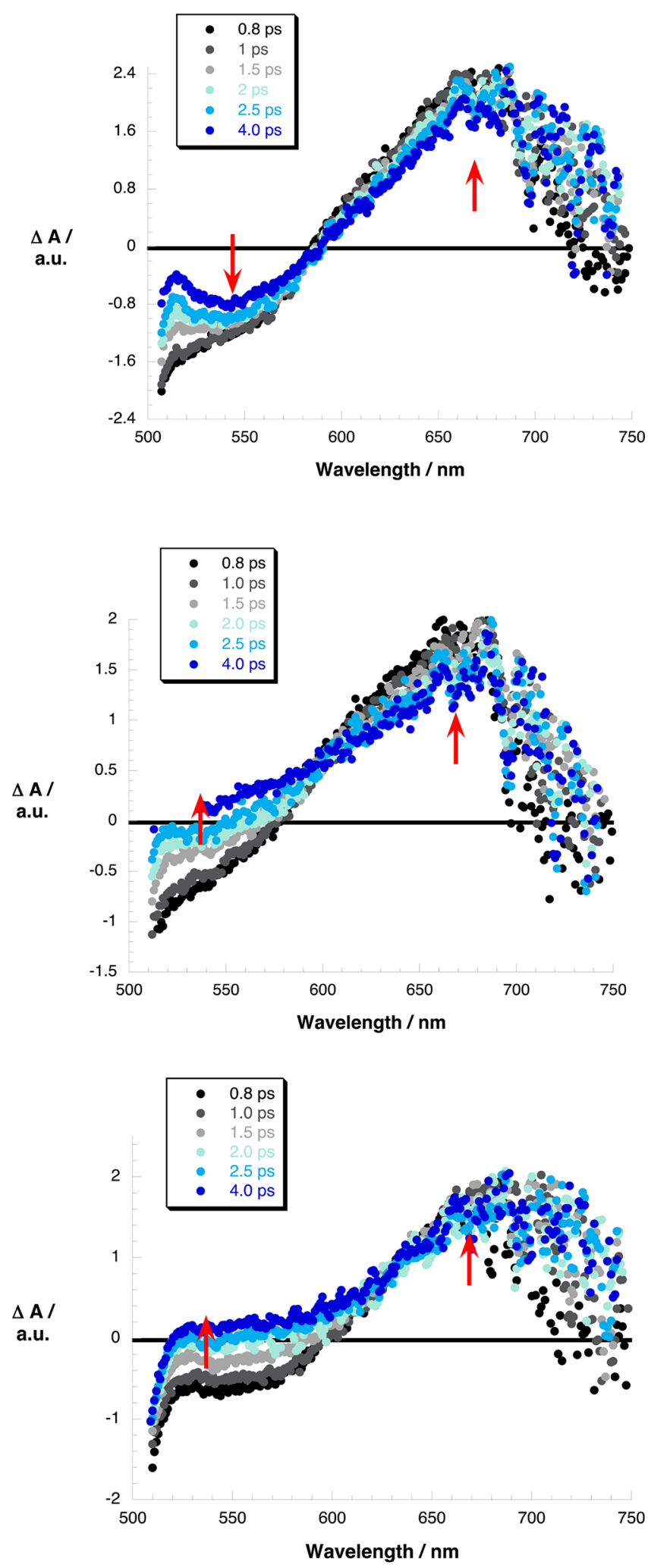

Figure 5. Femtosecond transient absorption $\left(\lambda_{\text {exc }}=480 \mathrm{~nm}\right)$ spectra of the formation of the radical cation in $0.1 \mathrm{mM}$ DMF solutions of SD1 (top), SD-2 (middle), and SD-5 (bottom) showing an accelerated recovery of the bleaching when going from SD-1 to SD-2 to SD-5..

Adsorption of the dyes on $\mathrm{Al}_{2} \mathrm{O}_{3}$ leads, in general, to an equal scenario, as found in the solution studies: the singlet excitation results in spectral signatures that are comparable but slightly broadened. The broadening on $\mathrm{Al}_{2} \mathrm{O}_{3}$ stems from the deprotonation of the cyanoacrylic acid and from intermolecular interactions, due to closer contacts between the dye molecules when adsorbed onto the $\mathrm{Al}_{2} \mathrm{O}_{3}$ surface, which results in a delocalization and thus stabilization of the radical cation. As a consequence, the dependence on the position of the inserted thiophene is relativized, and the signal solely depends on the number of inserted thiophenes. In other words, SD-1 lacks the bleaching and exhibits a sharper TA cation signal, whereas the bleaching in SD-4 is red-shifted with a TA radical cation $\left(\mathrm{TA}^{\bullet+}\right)$ absorption from 540 to $750 \mathrm{~nm}$, as seen in Figure 6. The rate constants for the intramolecular charge separation $\left(k_{\mathrm{CS}}\right)$ and singlet deactivation $\left(k_{\mathrm{SD}}\right)$ are given in Table 1.

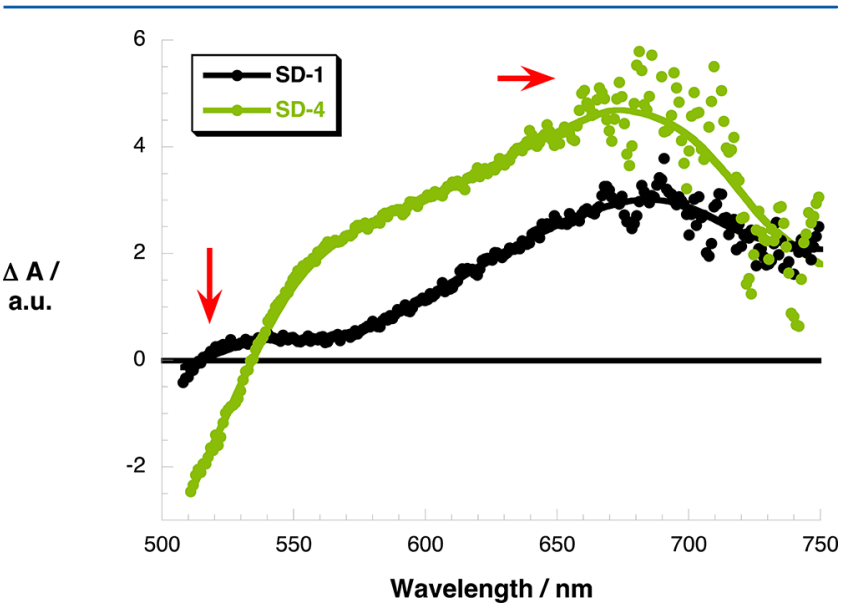

Figure 6. Transient absorption spectra showing the formation of the radical cation upon singlet excitation in $3 \mu \mathrm{m} \mathrm{Al}_{2} \mathrm{O}_{3}$ films of SD-1 (black) and SD-4 (green) resulting from femtosecond transient absorption studies at $480 \mathrm{~nm}$ excitation. The arrows indicate the corresponding signals as mentioned in the text.

The situation changes after adsorption onto $\mathrm{TiO}_{2}$. The deprotonation of the anchoring group upon binding and the electronic coupling to the $\mathrm{Ti}(3 \mathrm{~d})$ conduction band states of $\mathrm{TiO}_{2}$ lead to changes of the $\pi$-conjugated system of the dyes. Vibrational redistribution and solid-state interactions between the dye molecules and $\mathrm{TiO}_{2}$ result in broad positive bands, which correspond to the oxidized states of the dyes.

For all dyes, a broad maximum between 550 and $750 \mathrm{~nm}$ develops on the time scale of $<1$ ps (Figure 7). This corresponds to extremely fast charge injection into $\mathrm{TiO}_{2}$ with injection rates, as listed in Table 1 . Thereby, the chargeinjection rates were found to depend on the chemical structure of the dyes. In other words, the differences in $\pi$-conjugation and the variation of the donor-acceptor distances within the dye series impact the charge injection into $\mathrm{TiO}_{2}$ in a similar fashion as they influence the charge separation in solution. In this regard, considering the results of our theoretical investigation (see above) provides further understanding. Thus, inserting the thiophenes results in a localization of the LUMOs, which in turn changes the electronic coupling into $\mathrm{TiO}_{2}$ and increases the distance between the positively charged oxidized TA center and the $\mathrm{TiO}_{2}$ surface. Therefore, the fastest charge injection is expected for SD-1 and the slowest is expected for SD-5. Certainly, the increasing donor-acceptor distance plays a major role in the retardation of the chargeinjection rates from SD-1 to SD-2 to SD-5. Thus, the energetically low-lying and localized LUMO and the large donor-acceptor distance in SD-5 decrease the driving force for 
Table 1. Charge Separation/Charge Injection $\left(k_{\mathrm{CS}} / k_{\mathrm{CI}}\right)$, Singlet Deactivation $\left(k_{\mathrm{SD}}\right)$, and Direct Recombination Rate Constants

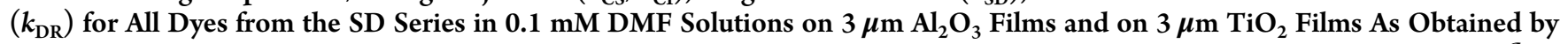
Femtosecond Transient Absorption Measurements with $480 \mathrm{~nm}$ Light Excitation at High Charge-Carrier Concentrations ${ }^{a}$

\begin{tabular}{|c|c|c|c|c|c|}
\hline & SD-1 & SD-2 & SD-3 & SD-4 & $\mathrm{SD}-5$ \\
\hline \multicolumn{6}{|l|}{ DMF } \\
\hline$k_{\mathrm{CS}} / \mathrm{s}^{-1}$ & $6.2 \pm 1.6 \times 10^{12}$ & $5.1 \pm 1.1 \times 10^{12}$ & $6.0 \pm 1.7 \times 10^{12}$ & $5.2 \pm 1.4 \times 10^{12}$ & $4.6 \pm 1.4 \times 10^{12}$ \\
\hline$k_{\mathrm{SD}} / \mathrm{s}^{-1}$ & $9.79 \times 10^{9}$ & $1.01 \times 10^{10}$ & $1.69 \times 10^{10}$ & $1.19 \times 10^{10}$ & $5.31 \times 10^{9}$ \\
\hline \multicolumn{6}{|l|}{$\mathrm{Al}_{2} \mathrm{O}_{3}$} \\
\hline$k_{\mathrm{CS}} / \mathrm{s}^{-1}$ & $6.9 \pm 1.2 \times 10^{12}$ & $4.9 \pm 1.2 \times 10^{12}$ & $6.4 \pm 1.5 \times 10^{12}$ & $5.3 \pm 1.1 \times 10^{12}$ & $4.5 \pm 1.6 \times 10^{12}$ \\
\hline$k_{\mathrm{SD}} / \mathrm{s}^{-1}$ & $2.50 \times 10^{10}$ & $3.85 \times 10^{9}$ & $8.43 \times 10^{9}$ & $3.42 \times 10^{9}$ & $2.17 \times 10^{9}$ \\
\hline \multicolumn{6}{|l|}{$\mathrm{TiO}_{2}$} \\
\hline$k_{\mathrm{CI}} / \mathrm{s}^{-1}$ & $5.6 \pm 2.1 \times 10^{12}$ & $4.3 \pm 1.8 \times 10^{12}$ & $4.9 \pm 1.4 \times 10^{12}$ & $4.2 \pm 1.6 \times 10^{12}$ & $3.8 \pm 1.5 \times 10^{12}$ \\
\hline$K_{\mathrm{DR}} / \mathrm{s}^{-1}$ & $7.63 \times 10^{9}$ & $3.09 \times 10^{9}$ & $6.05 \times 10^{9}$ & $3.52 \times 10^{9}$ & $1.20 \times 10^{9}$ \\
\hline
\end{tabular}

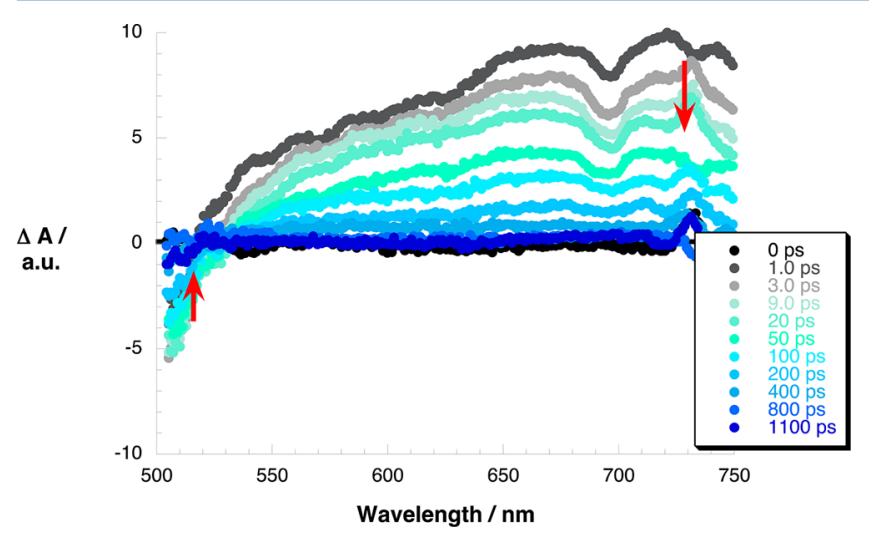

Figure 7. Transient absorption spectrum $\left(\lambda_{\mathrm{exc}}=480 \mathrm{~nm}\right)$ of SD-1 on $3 \mu \mathrm{m}$ mesoporous $\mathrm{TiO}_{2}$ with several time delays between 1 and 1100 ps. All dyes from the SD series show comparable spectral signatures.

charge injection, which lowers the rate constants in both directions.

The relatively high excitation intensity generates more than one electron per particle during one pulse. Because of the relatively short distance between the donor center and the anchoring group, most of the electrons recombine faster than the diffusion time in $\mathrm{TiO}_{2}$-intraparticle electron-hole recombination. ${ }^{16}$ Hence, under such conditions (high carrier concentrations) the signal of the oxidized state of the dyes decays on the time scale of $\sim 1$ ns - direct recombination (DR).

Two trends can be observed from the analysis of the corresponding decay dynamics, as found in the femtosecond transient absorption experiments. First, increasing the distance between the electron-donating and the electron-accepting moieties decelerates the DR from SD-1 to SD-2 and SD-5 (Figure 8). For SD-1, the curves reveal a monoexponential decay with shorter lifetimes than for SD-2 and SD-5, where the decay is biexponential with increasing lifetimes. Accordingly, in SD-2 and SD-5, the larger distances allow for a greater spatial separation of the positive and negative charge. This stabilizes the oxidized states of the dyes and leads to longer lifetimes.

Concerning SD-3 and SD-4, the insertion of thiophene into the donor seems to exhibit a lower impact on the DR from $\mathrm{TiO}_{2}$ than increasing the distance and, therefore, leads to comparable rate constants between SD-3 and SD-1 and between SD-4 and SD-2.

Hence, by changing the $\pi$-system of the dyes at different positions of the chemical structure, it is feasible to selectively influence either the light-harvesting properties or the charge-

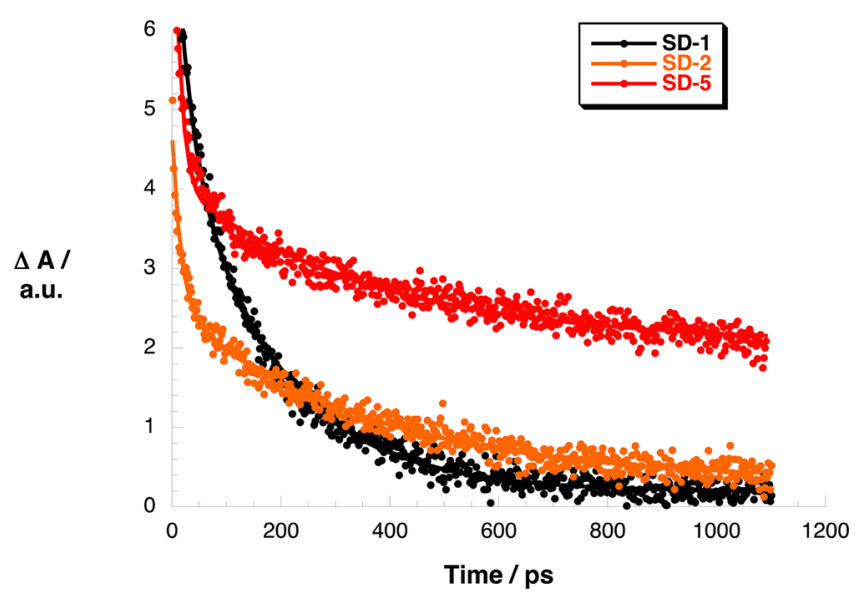

Figure 8. Time-absorption profiles at $650 \mathrm{~nm}$ as extracted from the transient absorption spectra $\left(\lambda_{\text {exc }}=480 \mathrm{~nm}\right)$ of SD-1 (black), SD-2 (orange), and SD-5 (red) on $3 \mu \mathrm{m}$ mesoporous $\mathrm{TiO}_{2}$ showing the change from the monoexponential decay in SD-1 to biexponential behavior in SD-2 and SD-5 and the increasing lifetimes.

transfer properties. Furthermore, the femtosecond studies show that under high charge-carrier concentration conditions the dynamics of the injection of electrons into $\mathrm{TiO}_{2}$ and their recombination correlate well with the dynamics of singlet excitation in solution and on $\mathrm{Al}_{2} \mathrm{O}_{3}$. Assuming that singlet excitation results from $\pi-\pi^{*}$ HOMO to LUMO transitions, it is directly linked to the $\pi$-system of the dyes. In other words, structural modifications, which affect the $\pi$-conjugation, have a direct effect on the charge transfer into $\mathrm{TiO}_{2}$ in such organic D$\pi$-A dyes.

To observe the charge recombination between single electrons injected into $\mathrm{TiO}_{2}$ and the oxidized states of the dyes we have employed nanosecond flash photolysis measurements. Thereby, the excitation intensities were kept at a much lower level than in the corresponding femtosecond studies so that per pulse less than one electron was injected into one nanoparticle. Under these conditions hole trapping can compete with charge-carrier recombination. In the trapped state, the hole is relatively unreactive toward electrons, which survive in the particles for many microseconds. ${ }^{16}$ This allows for probing the regeneration of the dyes' ground state by backelectron transfer from $\mathrm{TiO}_{2}$. Again, the spectral signature of $\mathrm{TA}^{\bullet+}$ can be used as a probe for the oxidized states of the dyes. Therefore, we have monitored the decays of the signals at 750 and $900 \mathrm{~nm}$ (Figure 9). 


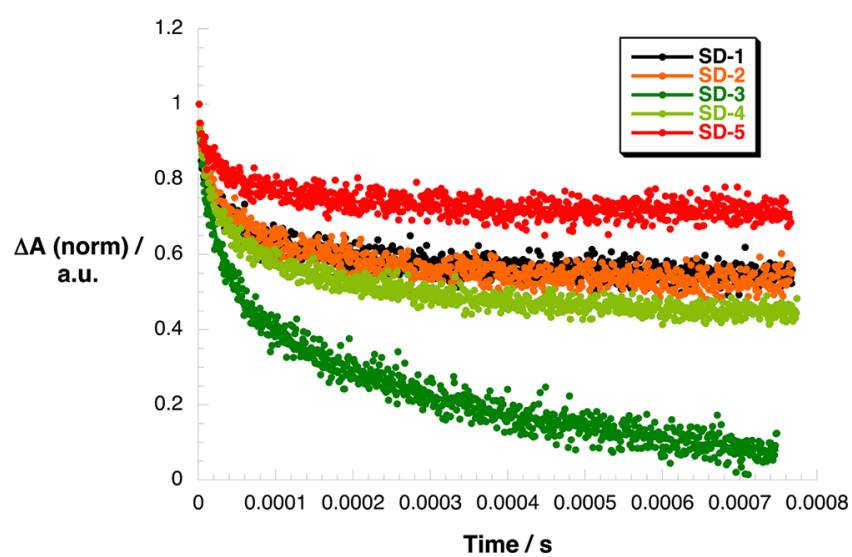

Figure 9. Normalized time-absorption profiles at $900 \mathrm{~nm}$ as extracted from the nanosecond flash photolysis spectra $\left(\lambda_{\text {exc }}=480 \mathrm{~nm}\right)$ of all dyes from the SD series on $3 \mu \mathrm{m}$ mesoporous $\mathrm{TiO}_{2}$ showing the different decay dynamics at low charge-carrier concentrations.

Biexponential fitting of the time absorption profiles at these wavelengths gave rise to two different rate constants (Table 2). Commonly, non-single exponential kinetics are a result of back electron transfer from trap states at different distances from the adsorbate with different trap energies. Electrons in deep trap states and at longer distances recombine more slowly with the adsorbate due to a smaller electronic coupling matrix element. ${ }^{17}$ Furthermore, the electronic coupling matrix element is governed by two other factors, namely, the energy and electron density distribution of the frontier orbitals on the anchoring parts of the dyes and the distance between the oxidized TA center and the $\mathrm{TiO}_{2}$ surface. In general, the stronger the electronic coupling between the electron and the hole the faster the recombination kinetics. As seen from Figure 9 and Table 2, both components of the charge recombination rate constants $\left(k_{\mathrm{CR} 1}\right.$ and $\left.k_{\mathrm{CR} 2}\right)$ show comparable dependencies on the dye structures. For SD-3 and SD-5, the relative contributions of the slow component $k_{\mathrm{CR} 2}$ to the overall decay behavior vary significantly. In SD-3, the decay kinetics are mainly dominated by the faster component $k_{\mathrm{CR} 1}$. In SD-5, the recombination occurs with the slower dynamics of $k_{\mathrm{CR} 2}$.

Because of equal anchoring groups in all dyes and therefore comparable electronic coupling of the anchor to $\mathrm{TiO}_{2}$, one would expect that the back electron transfer mainly depends on the charge-separation distance between the oxidized TA center and the $\mathrm{TiO}_{2}$ surface or, in other words, on the length of the spacer between donor and acceptor. This is, however, not the case because the electronic structure of the dyes - the spatial distribution of HOMOs and LUMOs and their energies changes significantly with the insertion of thiophenes at different positions of the dye architecture (see Molecular Modeling). Hence, the electronic coupling matrix element depends on the sum of the different contributions, that is, the anchoring moiety, the HOMO/LUMO distribution, and the separation distance between the electron and the hole.

As shown in Table 2, SD-1, SD-2, and SD-4 exhibit comparable back electron transfer kinetics, which are faster than for SD-3 and SD-5. The similar donor-acceptor distance and the comparable HOMO/LUMO distribution are responsible for this trend in SD-2 and SD-4 (see Figure 3). The energetically low-lying LUMOs, which are localized on the anchoring part of SD-2 and SD-4, provide strong electronic coupling to $\mathrm{TiO}_{2}$, which facilitates the recombination even from deeper trap states. Hence, the insertion of one thiophene results in a localization of the LUMO on the anchor and favors the electronic coupling between the dye and $\mathrm{TiO}_{2}$. As a consequence, the recombination occurs on a similar time scale as for the shorter SD-1, where HOMO and LUMO are conjugated throughout the entire molecular structure. In SD-3, one would expect a similar recombination behavior as for SD-1 due to a comparable electronic structure and equal donoracceptor distances. The extension of the $\pi$-system in the electron-donating part of SD-3, however, allows for a better spatial distribution of the positive charge, which stabilizes the oxidized state in energy and retards the back electron transfer as compared with SD-1. As seen from the LUMO in Figure 3, the electronic coupling of SD-3 into the $\mathrm{TiO}_{2}$ surface will be less efficient than for SD-2 or SD-4. Therefore, mainly states with low trap energies are stabilized and $k_{\mathrm{CR} 1}$ is the main component of the decay. Different is the situation in SD-5, where the strong localization of the LUMO on the anchor leads to good electronic coupling into the $\mathrm{TiO}_{2}$ and the recombination occurs from deeper trap states, which is reflected in the main contribution of $k_{\mathrm{CR} 2}$ for this process. The obviously slower rate constants in SD-5 result from the fact that apparently the increased distance between donor and acceptor comes into play and the back electron transfer becomes dependent on the separation distance between donor and acceptor.

To obtain further insight into this dependence of the rate constants on the distance, we analyzed the charge separation and recombination kinetics as a function of the distance between the TA center and the cyanoacrylic acid acceptor. This prompts to an exponential relationship $\left(k_{\mathrm{CT}}=k_{0} x\right.$ exp$\left.\left(-\beta r_{\mathrm{DA}}\right)\right),{ }^{18}$ which states that the electronic coupling between the donor and acceptor as mediated by the $\pi$-conjugated bridge decays exponentially with the separation length between the donor and the acceptor $\left(r_{\mathrm{DA}}\right)$. Accordingly, the slopes of the linear fits (Figure S2 of the Supporting Information) of the logarithmic plots of the charge-transfer rate constants $\left(k_{\mathrm{CT}}\right)$ as a function of donor-acceptor distance give rise to the so-called attenuation factor $\beta$. Applying this relationship to the SD dye series yielded different $\beta$ values for charge separation and charge recombination in solution and on $\mathrm{TiO}_{2}$.

As shown above, photoexcitation in solution results in a charge shift from the TA localized HOMO to the cyano-acrylicacid-localized LUMO. This charge-separation process most

Table 2. Charge Recombination Rate Constants $\left(k_{\mathrm{CR}}\right)$ for All Dyes from the SD Series on $3 \mu \mathrm{m} \mathrm{TiO}_{2}$ Films As Obtained from Bi-Exponential Fitting of the Time-Absorption Curves from Nanosecond Laser Flash Photolysis Studies with $480 \mathrm{~nm}$ Light Excitation at Low Charge-Carrier Concentrations

$\begin{array}{lccccc} & \mathrm{SD}-1 & \mathrm{SD}-2 & \mathrm{SD}-3 & \mathrm{SD}-4 & \\ \mathrm{TiO}_{2} & & & & & \mathrm{SD}-5 \\ k_{\mathrm{CR} 1} / \mathrm{s}^{-1} & 5.9 \times 10^{4} & 5.2 \times 10^{4} & 4.0 \times 10^{4} & 5.9 \times 10^{4} & 1.9 \times 10^{4} \\ k_{\mathrm{CR} 2} / \mathrm{s}^{-1} & 5.2 \times 10^{3} & 6.0 \times 10^{3} & 3.0 \times 10^{3} & 5.3 \times 10^{3} & 2.1 \times 10^{2}\end{array}$


likely involves a charge transfer through the linker, which interconnects donor and acceptor. Because of the fully conjugated structure of the molecules it is safe to assume that those spacers are rather rigid and therefore may be treated as molecular wires. Applying the above-mentioned exponential relationship for the distance dependence of the electronic coupling mediated by $\pi$-conjugated spacers yields the attenuation factor $\beta$. In DMF, $\beta$ was determined to $0.08 \AA^{-1}$ for charge separation and $0.10 \AA^{-1}$ for charge recombination, which corresponds to values in the range of highly conjugated oligoene, oligoyne, and oligofluorene molecular wires ${ }^{19}$ and corroborates the highly $\pi$-conjugated structure of the SD dyes.

On $\mathrm{TiO}_{2}, \beta$ was determined to $0.01 \AA^{-1}$ for charge injection, which states that it is nearly independent of the distance because of the very efficient coupling of the anchoring group to the conduction band of $\mathrm{TiO}_{2}$. Efficient $\pi$-conjugation mediates this coupling effectively. For charge recombination, $\beta$ is 0.25 $\AA^{-1}$. This considerably higher value complies well with the fact that the electronic coupling becomes dependent on the separation distance between the donor and acceptor moieties. As shown above, the length of the spacer between the TA center and the anchoring group to a great extent governs the coupling. However, regarding the charge recombination, further factors such as the electronic structure of the dyes and the specific structure-dependent $\pi$-conjugation play a significant role, which might impact the back electron transfer mechanism.

In summary, the photophysical studies show that variations of the chemical structure and the $\pi$-conjugation strongly impact the charge -transfer properties of organic D- $\pi$-A dyes, which, at a first glance, seem to comprise comparable features. In fact, it turned out that not only the structural variations themselves but also their particular locality within the molecular architecture affect the photophysics.

\section{CONCLUSIONS}

In conclusion, we have investigated the photoinduced chargetransfer processes of a series of five analogous organic D- $\pi$-A dyes. In particular, two different modes of systematic extension of the dyes' $\pi$-system were examined by steady-state and timeresolved spectroscopy. Additionally, quantum-chemical methods provided insight into the electronic properties of the dyes. It was found that the two different substitution patterns, that is, substitution of the donor versus substitution of the $\pi$-spacer, lead to significant variations of the electronic structure of the investigated systems. It has been shown that singlet excitation both in solution and on $\mathrm{Al}_{2} \mathrm{O}_{3}$ as well as charge injection and recombination on $\mathrm{TiO}_{2}$ films not only depend on the $\pi$ conjugation length and the donor-acceptor distance but also depend on the specific alternation of electronic structure. Our studies reveal that the modifications of the $\pi$-system performed at different positions of the chemical architecture induce different effects on the charge-transfer properties. Thus, the insertion of thiophenes into the donor moiety directly affects the photoinduced absorption and the spectral characterstics of the excited states of SD-3 and SD-4. On the contrary, the insertion of the thiophenes into the spacer between donor and acceptor does not influence the spectral positions of the maxima and minima but leads to distance-dependent chargetransfer features in SD-1, SD-2, and SD-5.

The rate constants for charge separation and charge recombination have been analyzed under different conditions and correlated with the chemical structures of the dyes. Inter alia, this also allowed for the determination of the distance- dependence of the charge-transfer processes, which lead to attenuation factors $\beta$ in the range of 0.1 to $0.01 \AA^{-1}$.

\section{ASSOCIATED CONTENT}

\section{Supporting Information}

Experimental details of materials, instruments, and methods employed; absorption spectra on $\mathrm{TiO}_{2}$; representation of the dependence of the charge-separation $\left(\ln k_{\mathrm{CS}}\right)$ and charge recombination $\left(\ln k_{\mathrm{CR}}\right)$ rate constants on the donor-to-acceptor distances; and excited-state properties from TD-DFT calculations. This material is available free of charge via the Internet at http://pubs.acs.org.

\section{AUTHOR INFORMATION}

\section{Corresponding Author}

*E-mail: mateusz.wielopolski@epfl.ch (M.W.), je.moser@epfl. ch (J.-E.M.), and kykay@ajou.ac.kr (K-Y.K.).

\section{Notes}

The authors declare no competing financial interest.

\section{ACKNOWLEDGMENTS}

M.W. and J.-E.M. thank NCCR MUST, a research instrument of the Swiss National Science Foundation for generous support.

\section{REFERENCES}

(1) Grätzel, M. Recent Advances in Sensitized Mesoscopic Solar Cells. Acc. Chem. Res. 2009, 42, 1788-1798.

(2) Zollinger, H. Color Chemistry: Syntheses, Properties, and Applications of Organic Dyes and Pigments, 3rd ed.; VHCA and Wiley-VCH: Zürich and Weinheim, 2003.

(3) (a) Mishra, A.; Fischer, M. K. R.; Bäuerle, P. Metal-Free Organic Dyes for Dye-Sensitized Solar Cells: From Structure: Property Relationships to Design Rule. Angew. Chem., Int. Ed. 2009, 48, 2474-2499. (b) Imahori, H.; Umeyama, T.; Ito, S. Large $\pi$-Aromatic Molecules as Potential Sensitizers for Highly Efficient Dye-Sensitized Solar Cells. Acc. Chem. Res. 2009, 42, 1809-1818. (c) Wang, X.-F.; Tamiaki, H. Cyclic Tetrapyrrole Based Molecules for Dye-Sensitized Solar Cells. Energy Environ. Sci. 2010, 3, 94-106.

(4) (a) Marszalek, M.; Nagane, S.; Ichake, M.; Humphrey-Baker, R.; Paul, V.; Zakeeruddin, S. M.; Grätzel, M. Tuning Spectral Properties of Phenothiazine Based Donor-Pi-Acceptor Dyes for Efficient DyeSensitized Solar Cells. J. Mater. Chem. 2012, 22, 889-894. (b) Burfeindt, B.; Hannappel, T.; Storck, W.; Willig, F. Measurement of Temperature-Independent Femtosecond Interfacial Electron Transfer from an Anchored Molecular Electron Donor to a Semiconductor as Acceptor. J. Phys. Chem. 1996, 100, 16463-16465. (c) Sayama, K.; Tsukagoshi, S.; Hara, K.; Ohga, Y.; Shinpou, A.; Abe, Y.; Suga, S.; Arakawa, H. Photoelectrochemical Properties of J Aggregates of Benzothiazole Merocyanine Dyes on a Nanostructured TiO2 Film. J. Phys. Chem. B 2002, 106, 1363-1371.

(5) (a) Kim, J.-J.; Choi, H.; Lee, J.-W.; Kang, M.-S.; Song, K.; Kang, S. O.; Ko, J. A Polymer Gel Electrolyte to Achieve $\geq 6 \%$ Power Conversion Efficiency with a Novel Organic Dye Incorporating a LowBand-Gap Chromophore. J. Mater. Chem. 2008, 18, 5223-5229. (b) Mikroyannidis, J. A.; Suresh, P.; Roy, M. S.; Sharma, G. D. Triphenylamine- and Benzothiadiazole-Based Dyes with Multiple Acceptors for Application in Dye-Sensitized Solar Cells. J. Power Sources 2010, 195, 3002-3010. (c) Tang, Z.-M.; Lei, T.; Jiang, K.-J.; Song, Y.-L.; Pei, J. Benzothiadiazole Containing D- $\pi$-A Conjugated Compounds for Dye-Sensitized Solar Cells: Synthesis, Properties, and Photovoltaic Performances. Chem.-Asian J. 2010, 5, 1911-1917. (d) Velusamy, M.; Justin Thomas, K. R.; Lin, J. T.; Hsu, Y. C.; Ho, K. C. Organic Dyes Incorporating Low-Band-Gap Chromophores for Dye-Sensitized Solar Cells. Org. Lett. 2005, 7, 1899-1902. (e) Zhu, W.; Wu, Y.; Wang, S.; Li, W.; Li, X.; Chen, J.; Wang, Z.-S.; Tian, H. Organic D-A- $\pi$-A Solar Cell Sensitizers with Improved Stability and 
Spectral Response. Adv. Funct. Mater. 2011, 21, 756-763. (f) Kim, S.; Lim, H.; Kim, K.; Kim, C.; Kang, T.; Ko, M.; Park, N. Synthetic Strategy of Low-Bandgap Organic Sensitizers and Their Photoelectron Injection Characteristics. IEEE J. Sel. Top. Quantum Electron. 2010, 16, 1627-1634.

(6) (a) Katono, M.; Bessho, T.; Wielopolski, M.; Marszalek, M.; Moser, J.-E.; Humphry-Baker, R.; Zakeeruddin, S. M.; Grätzel, M. Influence of the Anchoring Modes on the Electronic and Photovoltaic Properties of D- $\pi-\mathrm{A}$ Dyes. J. Phys. Chem. C 2012, 116, 1687616884. (b) Katono, M.; Bessho, T.; Meng, S.; Humphry-Baker, R.; Rothenberger, G.; Zakeeruddin, S. M.; Kaxiras, E.; Grätzel, M. D-pi-A Dye System Containing Cyano-Benzoic Acid as Anchoring Group for Dye-Sensitized Solar Cells. Langmuir 2011, 27, 14248-14252.

(7) Haid, S.; Marszalek, M.; Mishra, A.; Wielopolski, M.; Teuscher, J.; Moser, J.-E.; Humphry-Baker, R; Zakeeruddin, S. M.; Grätzel, M.; Bäuerle, P. Significant Improvement of Dye-Sensitized Solar Cell Performance by Small Structural Modification in $\pi$-Conjugated DonorAcceptor Dyes. Adv. Funct. Mater. 2012, 22, 1291-1302.

(8) (a) Bauer, C.; Teuscher, J.; Pelet, S.; Wenger, B.; Bonhôte, P.; Nazeeruddin, M. K.; Zakeeruddin, S. M.; Comte, P.; Grätzel, M.; Moser, J.-E. Ultrafast Charge Transfer Through p-Oligo(phenylene) Bridges: Effect of Nonequilibrium Vibrations. Curr. Sci. 2010, 99, 343-352. (b) Wenger, B.; Bauer, C.; Nazeeruddin, M. K.; Comte, P.; Zakeerudin, S. M.; Grätzel, M.; Moser, J.-E. Electron Donor-Acceptor Distance Dependence of the Dynamics of Light-Induced Interfacial Charge Transfer in the Dye-Sensitization of Nanocrystalline Oxide Semiconductors. Proc. SPIE 2006, 6325, 63250V 1-11.

(9) Alibabaei, L.; Kim, J.-H.; Wang, M.; Pootrakulchote, N.; Teuscher, J.; Di Censo, D.; Humphry-Baker, R.; Moser, J.-E.; Yu, Y.J.; Kay, K.-Y. Molecular Design of Metal-Free D- $\pi$-A Substituted Sensitizers for Dye-Sensitized Solar Cells. Energy Environ. Sci. 2010, 3, $1757-1764$.

(10) Wang, Z. S.; Cui, Y.; Dan-oh, Y.; Kasada, C.; Shinpo, A.; Hara, $K$. Thiophene-Functionalized Coumarin Dye for Efficient DyeSensitized Solar Cells: Electron Lifetime Improved by Coadsorption of Deoxycholic Acid. J. Phys. Chem. C 2007, 111, 7224-7230.

(11) Zhao, Y.; Truhlar, D. G. The M06 Suite of Density Functionals for Main Group Thermochemistry, Thermochemical Kinetics, Noncovalent Interactions, Excited States, and Transition Elements: Two New Functionals and Systematic Testing of Four M06-Class Functionals and 12 Other Functionals. Theor. Chem. Acc. 2008, 120, 215-241.

(12) Rassolov, V. A.; Pople, J. A.; Ratner, M. A.; Windus, T. L. 6$31 \mathrm{G}^{*}$ basis set for atoms K through Zn. J. Chem. Phys. 1998, 109, $1223-1229$.

(13) Frisch, M. J., et al. Gaussian 09; Gaussian, Inc.: Wallingford, CT, 2009.

(14) Tomasi, J.; Mennucci, B.; Cammi, R. Quantum Mechanical Continuum Solvation Models. Chem. Rev. 2005, 105, 2999-3093.

(15) (a) Oyama, M.; Higuchi, T.; Okazaki, S. An Intermediate State of the Triphenylamine Cation Radical Revealed Using an ElectronTransfer Stopped-Flow Method. Electrochem. Solid-State Lett. 2002, 5, E1-E3. (b) Fantacci, S.; De Angelis, F.; Nazeeruddin, M. K.; Gratzel, M. Electronic and Optical Properties of the Spiro-MeOTAD Hole Conductor in Its Neutral and Oxidized Forms: A DFT/TDDFT Investigation. J. Phys. Chem.C 2011, 115, 23126-23133. (c) Pinzón, J. R.; Gasca, D.; Gayathri, S.; Bottari, G.; Torres, T.; Guldi, D. M.; Echegoyen, L. Photoinduced Charge Transfer and Electrochemical Properties of Triphenylamine Ih-Sc3N@C80 Donor-Acceptor Conjugates. J. Am. Chem. Soc. 2009, 131, 7727-7734.

(16) Rothenberger, G.; Moser, J.; Grätzel, M.; Serpone, N.; Sharma, D. K. Charge Carrier Trapping and Recombination Dynamics in Small Semiconductor Particles. J. Am. Chem. Soc. 1985, 107, 8054-8059.

(17) (a) Weng, Y.-X.; Wang, Y.-Q.; Asbury, J. B.; Ghosh, H. N.; Lian, T. Back Electron Transfer from $\mathrm{TiO}_{2}$ Nanoparticles to FeIII(CN)6/3-: Origin of Non-Single-Exponential and Particle Size Independent Dynamics. J. Phys. Chem. B 2000, 104, 93-104. (b) Lu, H.; Prieskorn, J. N.; Hupp, J. T. Fast Interfacial Electron Transfer: Evidence for Inverted Region Kinetic Behavior. J. Am. Chem. Soc. 1993, 115, 4927-
4928. (c) Vrachnou, E.; Vlachopoulos, N.; Graetzel, M. Efficient Visible Light Sensitization of Titanium Dioxide by Surface Complexation with $\mathrm{Fe}(\mathrm{CN}) 64$. J. Chem. Soc., Chem. Commun. 1987, 868-870.

(18) Molecular Wires. From Design to Properties; De Cola, L., Ed.; Topics in Current Chemistry 257; Springer: Berlin, 2005; pp 63-103.

(19) (a) Benniston, A. C.; Goulle, V.; Harriman, A.; Lehn, J.-M.; Marczinke, B. Electron Delocalization in Polyene-Bridged Binuclear Complexes. J. Phys. Chem. A 1994, 98, 7798-7804. (b) Osuka, A.; Tanabe, N.; Kawabata, S.; Speiser, I. S. Tandem Reactions in Organic Synthesis: Novel Strategies for Natural Product Elaboration and the Development of New Synthetic Methodology. Chem. Rev. 1996, 96, 195-206. (c) Wielopolski, M.; Rojas, G.; van der Pol, C.; Brinkhaus, L.; Katsukis, G.; Bryce, M. R.; Clark, T.; Guldi, D. M. Control Over Charge Transfer Through Molecular Wires by Temperature and Chemical Structure Modifications. ACS Nano 2010, 4, 6449-6462. (d) Walther, M. E.; Grilj, J.; Hanss, D.; Vauthey, E.; Wenger, O. S. Photoinduced Processes in Fluorene-Bridged Rhenium-Phenothiazine Dyads - Comparison of Electron Transfer Across Fluorene, Phenylene, and Xylene Bridges. Eur. J. Inorg. Chem. 2010, 4843-4850. 